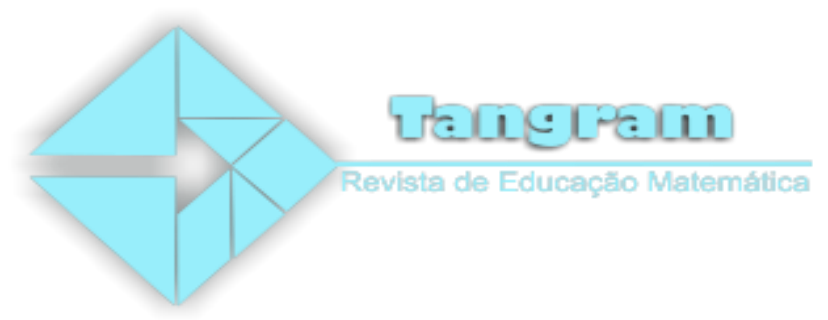

\title{
Enseñanza de estocásticos a niños con discapacidad. Un acercamiento epistemológico
}

\section{Teaching stochastics to children with disabilities. An epistemological approach}

\author{
J. Marcos López-Mojica ${ }^{1}$ \\ Catalina Navarro Sandoval ${ }^{2}$
}

\begin{abstract}
Resumen. En el presente documento se informa sobre una experiencia de enseñanza al introducir los temas de probabilidad y de estadística a niños con discapacidad del segundo grado (8-10 años) de educación especial. El interés fue documentar el proceso de enseñanza y, con base en ello, analizar el desempeño de los estudiantes ante una situación aleatoria. Para lo anterior fueron necesarios los elementos: epistemológico, cognitivo y social. Se aplicó una actividad de enseñanza para promover la comprensión de ideas fundamentales de estocásticos ante la presencia de la docente titular y el investigador. Los resultados muestran un acercamiento a nociones de espacio muestra, medida de probabilidad y variable aleatoria (frecuencias relativas). Se concluye necesario e imperante el tratamiento de fenómenos aleatorios en este nivel educativo de manera sistemática para ofrecer una matemática básica integral; además los estocásticos provocaron dotar de sentido a otros conceptos matemáticos por su uso.
\end{abstract}

Palabras clave: matemáticas escolares; probabilidad; estadística; discapacidad.

\begin{abstract}
The present document reports on an experience in the introduction of stochastics (concepts related to probability and statistics) to children with disabilities in the second grade (6-8 years) of special education. The interest was to document the teaching process and analyze the performance, qualitatively, of students in a random situation. For the above, the elements were necessary: epistemological, cognitive and social. A teaching activity was applied to promote the understanding of fundamental stochastic ideas in the presence of the head teacher and the researcher. The results show an approach to notions of sample space, probability measure and random variable (relative frequencies). It is concluded necessary and prevailing the treatment of random phenomena in this educational level in a systematic way to offer an integral basic mathematics; stochastics also gave rise to meaning to other mathematical concepts by their use.
\end{abstract}

Key words: school mathematics; probability; statistics; disabilities.

\footnotetext{
${ }^{1}$ Profesor Investigador, Facultad de Matemáticas, Universidad Autónoma de Guerrero

${ }^{2}$ Investigadora Titular.Posgrado en Matemática Educativa.Facultad de Matemáticas.Universidad Autónoma de Guerrero
}

Tangram - Revista de Educação Matemática, Dourados - MS - v.2 n. 2, pp. 87 - 101 (2019) 
Enseñanza de estocásticos a niños con discapacidad. Un acercamiento epistemológico

\section{Introducción}

En educación matemática, Bruno, Noda, Aguilar, González, Moreno y Muñoz (2006), expresan que el año 2000 fue declarado año mundial de las matemáticas, lo cual propició a la reflexión de "cómo poner la matemática al alcance de los niños con necesidades educativas especiales, en particular los que presentan alguna discapacidad" (p. 212). A casi 18 años sigue vigente esa reflexión, más aún existe la carencia de investigaciones que se interesen por los procesos cognitivos o estrategias de enseñanza que permitan el desarrollo de un pensamiento matemático de esta población. Así como de la formación del docente de educación especial en los temas matemáticos.

Por otra parte, se ha mostrado la problemática de desatención que, en general priva en la enseñanza de estocásticos en los distintos niveles educativos. Por lo que se conjetura extensiva a la educación especial. Particularmente, la ausencia de los temas de probabilidad y de estadística, dificulta identificar aspectos importantes del pensamiento probabilístico a tomar en cuenta en el planteamiento de actividades que permitan la introducción de la probabilidad en el sistema educativo mexicano.

El descuido de la formación del pensamiento probabilístico de los estudiantes, en cualquier nivel educativo, ha favorecido la desatención a formarlos integralmente. Lo anterior ha generado una sociedad determinista. El planteamiento de formación integral refiere a preparar a los alumnos para enfrentar situaciones deterministas como indeterministas.

Así, para el caso de este estudio, es de interés analizar las conductas manifiestas de los niños del segundo grado de educación especial durante una actividad sobre un fenómeno aleatorio, conductas que, en todo caso, serán efecto de las discapacidades particulares de que se trate y que permitirán diseñar actividades en función de las características de la población.

En ese sentido, Gaviria, Torres y Torres (2014) exponen la necesidad de herramientas didácticas que estén adaptadas a la población que presenta alguna discapacidad. Pues algunos profesores no están capacitados para el diseño y gestión de su clase con un enfoque inclusivo.

Tangram - Revista de Educação Matemática, Dourados - MS - v.2 n. 2, pp. 87-101 (2019) 


\section{Enseñanza de estocásticos a niños con discapacidad. Un acercamiento epistemológico}

Por lo tanto, la pregunta que rige a este escrito es ¿cuáles son los procesos de enseñanza de estocásticos (puestos en juego) en el segundo grado de educación especial? Se pretende caracterizar el proceso de enseñanza de los estocásticos en el nivel educativo en cuestión, para promover un marco de referencia que apoye a los docentes en su práctica de aula.

\section{El contexto de la educación especial}

En México, la educación especial es una modalidad educativa creada para atender a poblaciones que presentan necesidades educativas especiales asociadas o no a una discapacidad. La Ley General para las Personas con Discapacidad (LGPD, 2008) la refiere como un conjunto de servicios especializados puestos a disposición de las personas con discapacidad para lograr los fines de la educación.

Los servicios que ofrece la educación especial se agrupan en: de orientación, de apoyo y escolarizados. Dentro de los últimos se encuentran los Centros de Atención Múltiple (CAM), los cuales se encargan de escolarizar a niños que presentan alguna discapacidad o discapacidad múltiple, o bien aquéllos que, por otras circunstancias, no pueden ser integrados en escuelas regulares (Secretaría de Educación Pública, 2004), en este espacio fue donde se aplicó la estrategia de enseñanza.

Respecto a los niños que están incorporados a las aulas regulares, el plan de estudios considera la atención a la diversidad como un aspecto de la educación inclusiva. Ésta la refiere así "porque se ocupa de reducir al máximo la desigualdad del acceso a las oportunidades, y evita los distintos tipos de discriminación a las que están expuestas niñas, niños y adolescentes" (SEP, 2011; pág. 35). Particularmente, para los niños con discapacidad, el plan de estudios recomienda "estrategias de aprendizaje y enseñanza diferenciadas" (SEP, 2011; pág. 35), sin embargo no las define y tampoco expresa una manera de ponerlas en práctica.

En lo que respecta a la formación del docente de educación especial, ésta se desarrolla en ocho semestres. Su preparación concierne a: elementos generales de la educación básica, elementos de la formación para ser un docente de educación especial y una formación

Tangram - Revista de Educação Matemática, Dourados - MS - v.2 n. 2, pp. 87-101 (2019) 


\section{Enseñanza de estocásticos a niños con discapacidad. Un acercamiento epistemológico}

específica para brindar respuesta a las necesidades educativas especiales que presenten niños y adolescentes con una discapacidad (SEP, 2004).

Por otra parte, en el desarrollo integral, respecto a la educación de los niños con discapacidad, debería considerarse la preparación tanto para afrontar situaciones deterministas como situaciones bajo incertidumbre. Por lo que surge la interrogante de: ¿Cómo se prepara al niño con alguna ausencia o limitación para que enfrente situaciones indeterministas? La respuesta demanda la creación de un marco de referencia que permita a las docentes plantear actividades para introducir los temas en cuestión.

La inclusión de estocásticos en la educación requiere de investigaciones acerca de los procesos de su enseñanza y de la comprensión resultante de los educandos en la escuela regular u otros extractos de la educación, para tener un marco de referencia que permita caracterizar el tratamiento de estocásticos en cualquier nivel educativo y no sólo eso, sino extenderlo al campo profesional donde se requiera el uso de probabilidad y de estadística.

\section{Elementos teóricos}

Para poder analizar el proceso de enseñanza, se tomaron en consideración los siguientes elementos teóricos. Se partió de que la intuición se deriva de la experiencia (Fischbein, 1975) y de que surgen esquemas que compensan ausencias o limitaciones en el desarrollo de niños con deficiencias perceptuales, cognitivas o motoras (Vygotski, 1997). Por lo tanto, interesa centrar la atención en los elementos de orden epistemológico, cognitivo y social.

\section{Elementos epistemológicos}

La propuesta de Heitele (1975) sobre diez ideas fundamentales de estocásticos como guía para un curriculum en espiral es de importancia. Para el autor, una idea fundamental es “... aquella que proporciona al individuo modelos explicativos tan eficientes como sea posible" (pág. 188). Argumenta que el tratamiento de las ideas fundamentales debe partir de un plano intuitivo y arribar a un plano formal, de manera que se garantice continuidad en la educación.

Tangram - Revista de Educação Matemática, Dourados - MS - v.2 n. 2, pp. 87-101 (2019) 
Enseñanza de estocásticos a niños con discapacidad. Un acercamiento epistemológico

\section{Elementos cognitivos}

En su obra sobre las fuentes intuitivas del pensamiento probabilístico, Fischbein (1975) entiende por intuición un conocimiento que se deriva de la experiencia, de recuperación inmediata, sintético, que se extrapola y no susceptible de análisis. El autor señala que en la formación de intuiciones probabilísticas es necesario considerar lo incierto y conectarlo con la acción por medio de frecuencias relativas; de esta forma se establecerá un comportamiento de la situación aleatoria caracterizado como "más probable", "menos probable" o "igualmente probable". La probabilidad es, por tanto, apropiada para el estudio de esas intuiciones; debido a su enfoque frecuencial, la probabilidad está determinada por la acción y es en la acción u observancia de los fenómenos naturales como se puede desarrollar una base intuitiva.

Los esquemas compensatorios son procesos que permiten superar una ausencia o limitación, reestructurando la forma de ser del niño tal que asumen la función inactiva o dañada para lograr ese fin (Vygotski, 1997). El primer motor de desarrollo de la compensación es el hecho de que el niño se desenvuelve en un ambiente sociocultural diseñado para un humano normal.

\section{Eje social}

Steinbring (2005) establece una relación entre la naturaleza epistemológica del concepto matemático y su significado socialmente constituido en las interacciones en el aula. El autor argumenta que para la adquisición de un concepto matemático es necesaria la interacción entre el contexto de referencia en que se implica al objeto, el signo y el concepto matemático. La constitución del concepto resulta de un balance entre las relaciones entre los tres vértices, de modo que se pueda deducir el significado del conocimiento matemático.

\section{Método, instrumento y técnicas}

La experiencia siguió los lineamientos del órgano operativo y de la célula de análisis de la enseñanza (Ojeda, 2006). En el aula real de educación primaria especial se pusieron en juego los métodos de bitácora y de experienciación, con una actividad de enseñanza de estocásticos diseñadas ex profeso. Los instrumentos de registro de datos fueron los guiones

Tangram - Revista de Educação Matemática, Dourados - MS - v.2 n. 2, pp. 87-101 (2019) 
de estrategia de enseñanza y las hojas de control; las técnicas de registro de datos fueron la escritura con lápiz y papel, o en pizarrón, la videograbación y su transcripción.

Los criterios de análisis (Ojeda, 2006) fueron: ideas fundamentales de estocásticos, otros conceptos matemáticos, recursos semióticos, términos para referirse a estocásticos, esquemas compensatorios.

Participantes. El aula alterna (Ojeda, 2006) es una alternativa al aula tradicional. En la alterna confluyen la docencia y la investigación, de manera que la docente se inicia en la indagación de su propia enseñanza, de las relaciones entre el contenido matemático y las producciones de los niños. La actividad propuesta por el investigador en el aula alterna se dirigió a siete niños (8-10 años) con diversos niveles y tipos de afecciones (véase la Tabla 1) de un Centro de Atención Múltiple, institución que ofrece servicios educativos a niños con discapacidad. La actividad se aplicó en una sesión de 40 minutos en los tiempos institucionales establecidos.

Tabla 1. Características individuales en el aula alterna del segundo año de primaria.

\begin{tabular}{c|cccc|cc|c}
\hline & \multicolumn{3}{|c|}{ Síndrome de Down } & \multicolumn{2}{c|}{$\begin{array}{c}\text { Discapacidad } \\
\text { Intelectual }\end{array}$} & $\begin{array}{c}\text { Síndrom } \\
\text { e Weber }\end{array}$ \\
\hline \multirow{4}{*}{ Casos } & $\mathbf{B}$ & $\mathbf{E}$ & $\mathbf{J}$ & $\mathbf{C}$ & $\mathbf{I}$ & $\mathbf{K}$ & $\mathbf{M}$ \\
& Mujer & Hombre & Hombre & Hombre & Mujer & Mujer & Mujer \\
& 8 años & 8 años & 8 años & 11 años & 8 años & 11 años & 9 años \\
Lenguaj & Oralizad & Sonidos & Palabra & Sonidos & Palabra & Sonidos & \\
e & $\mathrm{a}$ & guturale & $\mathrm{s}$ & guturale & $\mathrm{s}$ & guturale & Oralizada \\
& & $\mathrm{s}$ & aisladas & $\mathrm{s}$ & aisladas & $\mathrm{s}$ & \\
\hline
\end{tabular}

La situación de referencia. La actividad de enseñanza "Calcula y gana" se deriva del libro de texto para el segundo grado de educación primaria regular (SEP, 2006, p. 32). De la aplicación de los criterios de análisis se identificaron las siguientes ideas fundamentales de estocásticos: independencia, pues el resultado del lanzamiento de un dado en nada afecta el resultado del lanzamiento del otro; espacio muestra, con la variación de resultados al lanzar los dos dados; variable aleatoria, de manera cualitativa con la frecuencia relativa de cada evento. La docente pidió a los niños y niñas la reproducción en una hoja

Tangram - Revista de Educação Matemática, Dourados - MS - v.2 n. 2, pp. 87-101 (2019) 
Enseñanza de estocásticos a niños con discapacidad. Un acercamiento epistemológico

cuadriculada de los datos de una tabla numerada del 1 al 100. La aplicación de los criterios de análisis se muestra en la siguiente tabla 2.

Tabla 2. Caracterización de la actividad (López-Mojica, Ojeda y Salcedo, 2018).

\begin{tabular}{|c|c|c|c|c|}
\hline $\begin{array}{c}\text { Situación y } \\
\text { contexto }\end{array}$ & $\begin{array}{c}\text { Ideas } \\
\text { fundamentales } \\
\text { de estocásticos }\end{array}$ & $\begin{array}{c}\text { Otros } \\
\text { conceptos } \\
\text { matemáticos }\end{array}$ & $\begin{array}{l}\text { Recursos } \\
\text { semióticos }\end{array}$ & $\begin{array}{l}\text { Términos } \\
\text { empleados }\end{array}$ \\
\hline $\begin{array}{l}\text { Juego con } \\
\text { lanzami-entos } \\
\text { sucesivos de } \\
\text { dos dados } \\
\text { distin-guibles; } \\
\text { asignación de } \\
\text { tantas decenas } \\
\text { como puntos se } \\
\text { obtengan con } \\
\text { uno de los } \\
\text { dados y de } \\
\text { tantas unidades } \\
\text { como puntos se } \\
\text { obtengan en el } \\
\text { otro. }\end{array}$ & $\begin{array}{l}\text { Espacio } \\
\text { muestra, } \\
\text { independencia, } \\
\text { una variable } \\
\text { aleatoria. }\end{array}$ & $\begin{array}{l}\text { Valor } \\
\text { posicional, } \\
\text { números } \\
\text { naturales, } \\
\text { orden, conteo, } \\
\text { adición y } \\
\text { sustracción. }\end{array}$ & $\begin{array}{l}\text { Lengua natural, } \\
\text { signos } \\
\text { numéricos, } \\
\text { gráfica, tablas, } \\
\text { figuras. }\end{array}$ & $\begin{array}{l}\text { Llegar a, } \\
\text { acertar, } \\
\text { avanzar, } \\
\text { avanzar hasta, } \\
\text { tirar, gana, } \\
\text { salieron, } \\
\text { cuántos. }\end{array}$ \\
\hline
\end{tabular}

La actividad consistió en trabajar en grupos utilizando dados distinguibles (azul y rojo) por el color y el tamaño, ordinarios (cúbicos, con puntos en las caras) (véase Figura 1). Se empleó una tabla para registrar el número de puntos obtenidos en cada lanzamiento, los dos dados se lanzaron consecutivamente; los puntos del dado azul indicaban unidades y los puntos del dado rojo indicaban decenas; se demandó la suma de los valores obtenidos y avanzar según el resultado de la suma, partiendo del valor inicial, en una cuadricula grande realizada por la docente para el registro en grupo. Gana quien arribe primero a la casilla 100.

Tangram - Revista de Educação Matemática, Dourados - MS - v.2 n. 2, pp. 87-101 (2019) 

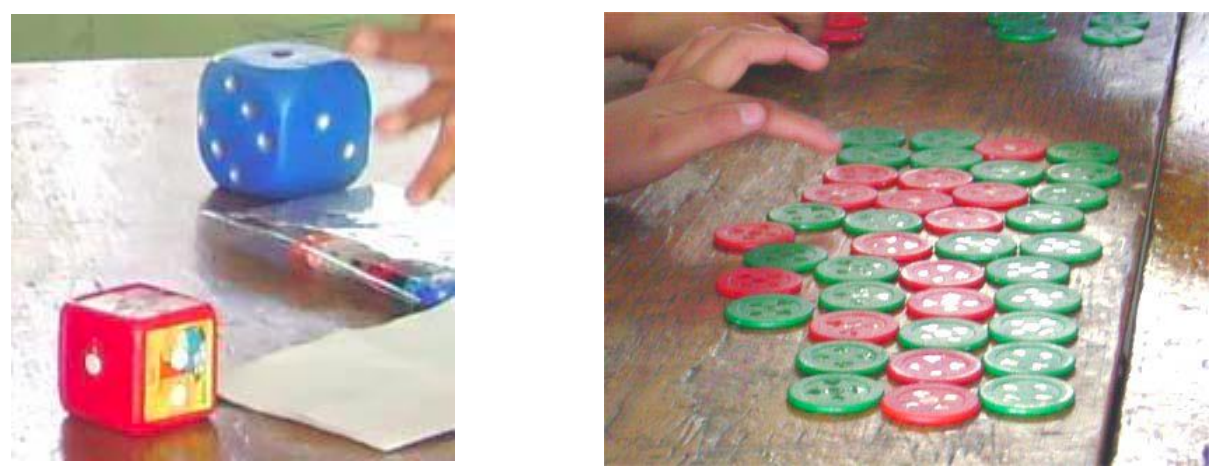

Figura 1. Material empleado en la actividad "Calcula y gana".

Los alumnos, para saber si llegaban o se aproximaban a 100, tenían que realizar la suma de las posiciones dadas y la obtenida mediante el conteo propuesto por la docente, utilizando fichas de dos colores, verdes y rojas, para las cantidades.

\section{Resultados de la enseñanza en el aula alterna}

En la actividad de enseñanza "Calcula y gana", dos niños con síndrome de Down (B y J) identificaron algunos casos del espacio muestra, K (discapacidad intelectual) y $\mathrm{M}$ (Síndrome Weber) asignaron valores a los puntos de los dados, lo cual permitió un acercamiento a la variable aleatoria. En las siguientes intervenciones, se emplea la inicial del nombre del alumno y $\mathbf{P}$ para la docente titular.

Espacio muestra. Se tuvo un acercamiento al espacio muestra al identificar algunos posibles resultados, por ejemplo, cuando la docente pedía que se lanzaran los dados, la pregunta “¿cuántas bolitas cayeron?” hizo centrar la atención del alumno en la cantidad de "puntitos" de la cara del dado que quedó hacia arriba; además, la pregunta orientó la acción del alumno a la asignación de un numeral respecto de la cantidad de "bolitas", de donde se aproximó al espacio muestra respectivo.

[110] ¿Cuántos cayeron? ¿Cuántas bolitas hay?

$\mathrm{P:}$

[111] I: ;Uno! [Gritando].

[112] Uno [sonriendo].

E:

Tangram - Revista de Educação Matemática, Dourados - MS - v.2 n. 2, pp. 87-101 (2019) 
Enseñanza de estocásticos a niños con discapacidad. Un acercamiento epistemológico

[113] Uno [levantando el dedo índice].

$\mathrm{P:}$

[385] ¡Cayó así! [Mostrando la cara del dado, 5]... ¿Cuánto

P: $\quad$ cayó?

[386] ¡Cinco! [Extendiendo la mano].

M:

[387] Cinco, ¿verdad?...

$\mathrm{P:}$

[388] K, M e ¡Sí!

I:

Independencia. Aunque se pudo haber introducido la idea de independencia en dos actividades, no se aprovechó la ocasión. Lo que se puede notar es que se identificó al fenómeno aleatorio $[94,96]$. Pero no ocurrieron expresiones del tipo "No importa lo que caiga en un dado, cualquiera de las seis caras pueden caer en el otro" que se refirieran a la idea de independencia:

[94] Estamos en éstos [señalando los números seleccionados

P: $\quad$ por los niños], con estos tres vamos [valores iniciales] a trabajar ¿si? Vamos a hacer lo posible por llegar al número cien. ¿Cómo lo vamos a hacer? Vamos a tirar nuestros dados...

[95] ¡Ay! [Molesta].

M:

[96] Si no llegamos a ese número, no importa, hasta donde

P: $\quad$ lleguemos. Vamos a tirar nuestro dado y lo vamos hacer; son cuatro [niños]. Lo vamos hacer por parejas; entonces, cada uno va a tirar, primero tira uno y después el otro, primero una pareja y después la otra; [refiriéndose a $\mathbf{M}$ ] ¿qué color quieres?...

Variable aleatoria. Con acciones como asignar valores numéricos a cada uno de los "puntitos de los dados" [8, 10], se sugiere el uso de nociones de esta idea fundamental.

Tangram - Revista de Educação Matemática, Dourados - MS - v.2 n. 2, pp. 87-101 (2019) 


\section{Enseñanza de estocásticos a niños con discapacidad. Un acercamiento epistemológico}

Nótese que la docente desconoció que se trata de dados ordinarios e incluyó valores que son imposibles de obtener: $0,7,8,9$ en un solo lanzamiento [6]; en consecuencia, el espacio muestra quedó desdibujado. Ningún niño se percató de esto. No obstante, los dígitos se utilizaron cuando se contaban las fichas.

[6] P: Decena significa que vamos a tener grupos de diez cosas; no importan las cosas que tengamos: perros, gatos, fichas, lápices. ¡No importa!, pero van a ser (...). El dado rojo [tomándolo en las manos] nos va a indicar el grupo de los dieces, el grupo de la decena; y éste [señalando el dado azul] nos va a indicar el grupo de las unidades, del uno al nueve, del cero al nueve, ehhh... Recuerden que cada una de las bolitas va a valer uno en este dado azul; ;uno! A ver, todos: ¡uno! [Moviendo el dedo índice].

[7] T: ¡Uno! [Moviendo el dedo índice de la mano derecha].

[8] P: ... Ehhh, cada bolita corresponde a uno. En este dado [tomando el dado rojo], que es el grupo de los dieces, el grupo de la decena, [cada una de las bolitas] va a valer diez, ¡así! [mostrando todos los dedos de ambas manos].

[9] T: ¡Diez! [Levantando las manos y agitándolas].

[10] ... Diez cosas, pero cada una de las bolitas va a valer diez, cada una

P: $\quad$ de las bolitas vale diez, ¿de acuerdo?

Se utilizaron soportes concretos (fichas) para los valores de la variable aleatoria (véase Figura 2). Un aspecto que hubiera convenido tomar en cuenta es la correspondencia de color entre dados y fichas para promover el uso de esquemas compensatorios del alumno.
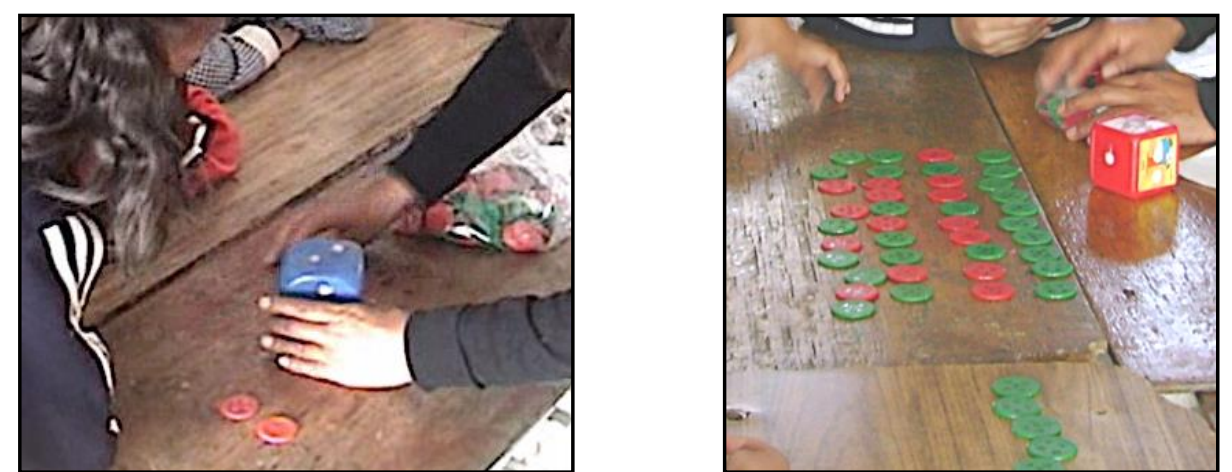

Figura 2. Asignación de fichas según los "puntitos" de cada dado.

Tangram - Revista de Educação Matemática, Dourados - MS - v.2 n. 2, pp. 87-101 (2019) 


\section{Enseñanza de estocásticos a niños con discapacidad. Un acercamiento epistemológico}

Valor posicional. En la actividad los "puntitos" de las caras de dos dados distinguibles indicaban, para uno, tantas unidades como puntos en la cara y, para el otro, tantas decenas como puntos en la cara. La docente centró su enseñanza en este tema, y no aludió al aspecto aleatorio que supone el material concreto utilizado $[6,8,10]$ :

[6] P: Decena significa que vamos a tener grupos de diez cosas; no importan las cosas que tengamos: perros, gatos, fichas, lápices. No importa, pero van a ser (...) El dado rojo [tomándolo en las manos] nos va a indicar el grupo de los dieces, el grupo de la decena; y éste [señalando el dado azul] nos va a indicar el grupo de las unidades, del uno al nueve, del cero al nueve, ehhh... recuerden que cada una de las bolitas va a valer uno en este dado azul; ¡uno! A ver, todos: ¡uno! [moviendo el dedo índice].

[7] T: ¡Uno! [Moviendo el dedo índice de la mano derecha].

[8] P: ... ehhh, cada bolita corresponde a uno; en este dado [tomando el dado rojo], que es el grupo de los dieces, el grupo de la decena, va a valer diez, ¡así! [moviendo los dedos de ambas manos].

[9] T: ¡Diez! [Levantando las manos y agitándolas].

[10] ... Diez cosas, pero cada una de las bolitas va a valer diez, cada una de P: $\quad$ las bolitas vale diez, ¿de acuerdo?

Adición de números naturales. De manera explícita, la docente agregó a una colección de fichas en cantidad correspondiente a una cifra dada, la obtenida del lanzamiento de los dados y pidió a los niños indicar la cantidad total de fichas (véase Figura 3). La docente indicó sumar, juntar todas las fichas a manera de estimular la adición.

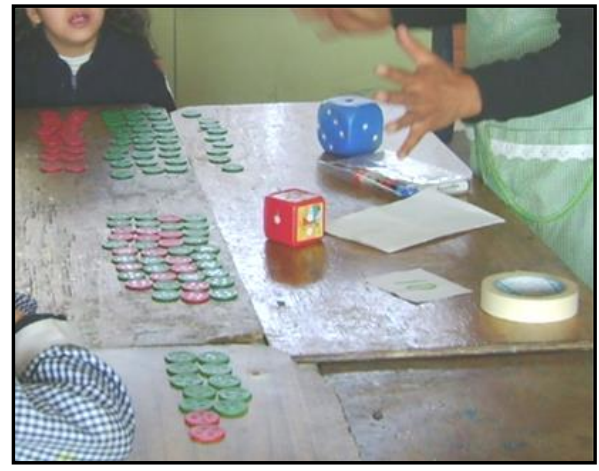

Figura 3. Agregación de dos colecciones de fichas y la cantidad total obtenida. 
Constitución del concepto y red conceptual. La actividad desarrollada en el aula alterna, sin pretenderlo, distinguió entre signo, objeto y concepto, lo cual es necesario para la constitución del concepto (Steinbring, 2005), en particular de estocásticos.

Situación de referencia (Objeto):

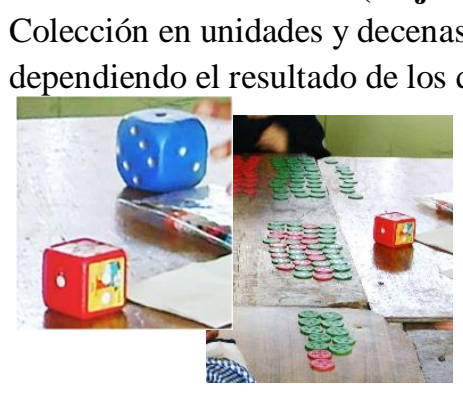

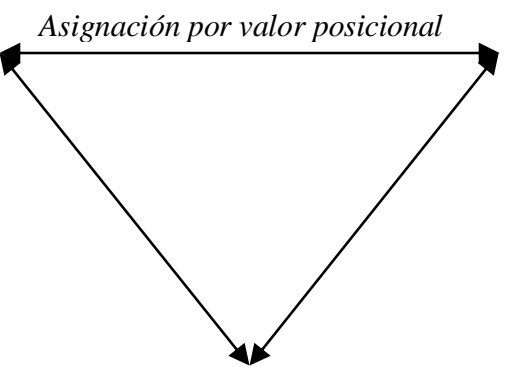

Concepto:

Variable Aleatoria
Signo: Numerales

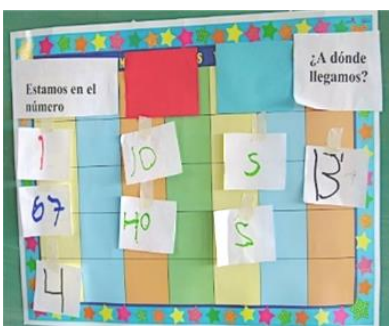

Figura 4. Triángulo epistemológico para el concepto de variable aleatoria (Steinbring, 2005).

Las estrategias de la docente permitieron dicha distinción. Para el caso del concepto de variable aleatoria, cuando al lanzar los dados ordinarios se asignó un numeral a la cantidad de "puntos" que tenía la cara del dado; para el signo, se utilizó una tabla, donde se anotaban y organizaban los resultados de las asignaciones, es decir, los valores de la variable aleatoria (ver Figura 4).

También se tuvo esa distinción para el espacio muestra, ya que las caras de los dados indicaban los posibles resultados del fenómeno aleatorio del lanzamiento de dados. Los numerales se utilizaron para identificar la cantidad de puntos de cada cara del dado (ver Figura 5).

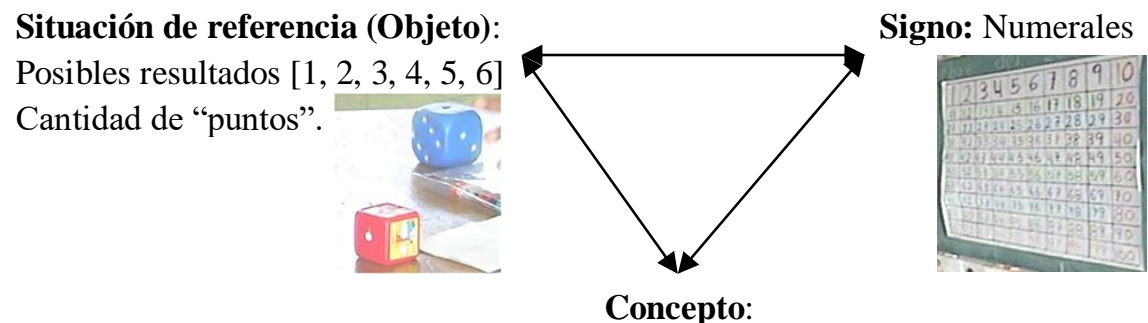

Concepto:

Espacio muestra

Figura 5. Triángulo epistemológico para el concepto de espacio muestra (Steinbring, 2005).

Tangram - Revista de Educação Matemática, Dourados - MS - v.2 n. 2, pp. 87-101 (2019) 
También se pudo identificar una red conceptual (Steinbring, 2005) promovida por la situación aleatoria desarrollada en el aula. Es decir, con el vértice objeto del triángulo epistemológico del concepto variable aleatoria (Figura 4), se pudieron desprender los vértices signo y concepto para constituir el valor posicional (ver Figura 6). Esto es, con la situación de referencia que consistió en la constitución de cantidades de dos cifras utilizando dados y fichas, para formar colecciones de unidades y decenas; y como signo se utilizó una tabla cuadriculada numérica con la serie del 1 al 100 (véase Figura 6).

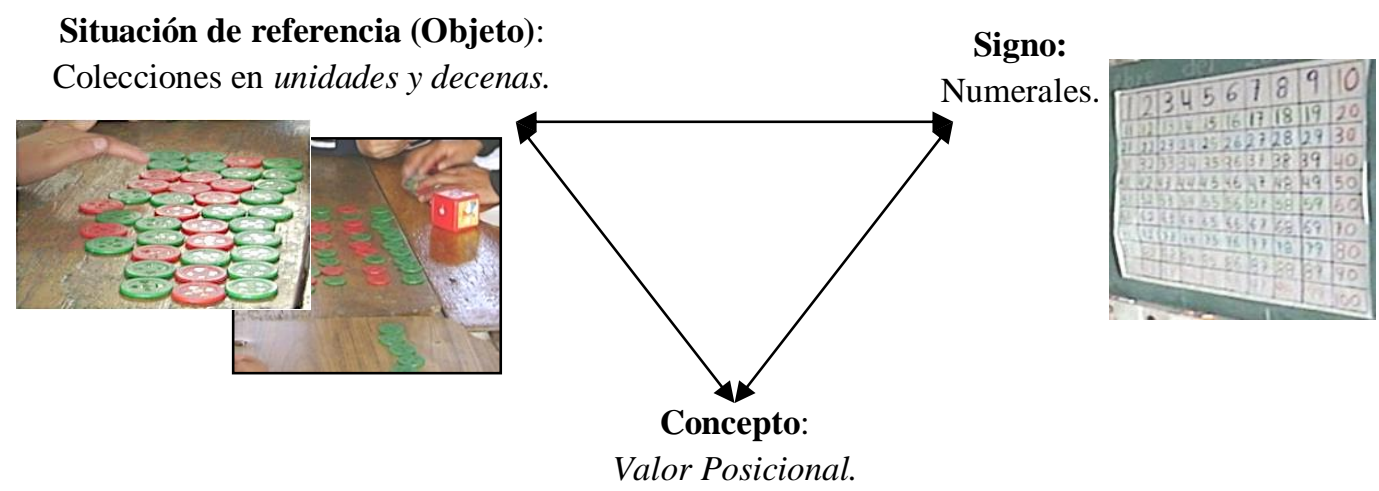

Figura 6. Triángulo epistemológico para el concepto de valor posicional (Steinbring, 2005).

La red conceptual permitió otorgarle otro sentido al concepto valor posicional por el uso en la actividad de referencia (Steinbring, 2005). Como se puede notar, los conceptos de estocásticos no deben deducirse de conceptos elementales, pues a su vez, éstos requieren de otros conceptos más básicos, desarrollándose una red de triángulos epistemológicos interconectados por uno de sus vértices (Steinbring, 2005; López-Mojica, Ojeda y Salcedo, 2018).

\section{Conclusiones y comentarios}

De los resultados se puede argumentar que, de los siete niños del segundo grado de primaria especial de un Centro de Atención Múltiple, en condiciones de síndrome de Down, síndrome Weber y discapacidad intelectual, se permite recomendar la enseñanza de la probabilidad y de la estadística en ese nivel educativo.

Tangram - Revista de Educação Matemática, Dourados - MS - v.2 n. 2, pp. 87-101 (2019) 


\section{Enseñanza de estocásticos a niños con discapacidad. Un acercamiento epistemológico}

Durante el desarrollo de la actividad se evidenció un acercamiento a las nociones de espacio muestra, medida de probabilidad y asignación de valores de algunos eventos por parte de los niños con discapacidad intelectual y síndrome de Down.

La promoción en actividades de enseñanza de experiencias con situaciones que impliquen fenómenos aleatorios, favorecen el desarrollo de intuiciones (Fischbein, 1975). Entre más experiencias sobre estocásticos tengan los niños desde edades tempranas, más se fomentará el desarrollo de las ideas fundamentales (Heitele, 1975) y se prevendrán los sesgos en el pensamiento probabilístico (Tversky y Kahneman, 1982).

Lo anterior cumple también para la población de educación especial, ya que de esa manera se le ofrece una educación integral. Además, los estocásticos son promotores de una red conceptual, es decir, convocan otros conceptos matemáticos como en este caso el valor posicional (Steinbring, 2005; López-Mojica, Ojeda y Salcedo, 2018).

No sólo es posible el tratamiento de la probabilidad en la educación especial, sino necesario para proporcionar una formación matemática integral, ésta debería de promover el uso de los esquemas compensatorios, pues ellos permiten desarrollar el pensamiento de los niños con discapacidad.

\section{Referências}

Bruno, A., Noda, M., Aguilar, R., González, C., Moreno, L. y Muñoz, V. (2006). Análisis de un tutorial inteligente sobre conceptos lógico-matemáticos en alumnos con síndrome de Down. Revista Latinoamericana en Matemática Educativa, 9(2), 211226.

Fischbein, E. (1975). The intuitive sources of probabilistic thinking in children. Holanda: Reidel.

Gaviria, Y. R., Torres, J. J., y Torres, E. (2014). Una propuesta inclusiva para la representación geométrica de los poliedros con población en condición de discapacidad visual. Infancias Imágenes, 13(2), 111-125. DOI: https://doi.org/10.14483/udistrital.jour.infimg.2014.2.a09

Tangram - Revista de Educação Matemática, Dourados - MS - v.2 n. 2, pp. 87-101 (2019) 


\section{Enseñanza de estocásticos a niños con discapacidad. Un acercamiento epistemológico}

Heitele, D. (1975). An Epistemological View on Fundamental Stochastic Ideas. Educational Studies in Mathematics 6(2), 187-205.

Ley General para las Personas con Discapacidad (2008, 1 de agosto). Diario Oficial de la Federación, México.

López-Mojica, J. M., Ojeda, A. M. y Salcedo, J. (2018). Ideas fundamentales de estocásticos en libros de texto de educación primaria: una alternativa de enseñanza. IE Revista de Investigación Educativa de la REDIECH, 9(17), 87-102.

Ojeda, A. M. (2006). Estrategia para un perfil nuevo de docencia: un ensayo en la enseñanza de estocásticos. En Filloy (Ed.) Matemática Educativa, treinta años (pp. 195-214). México: Santillana-Cinvestav.

Secretaría de Educación Pública (2004). Licenciatura en Educación Especial. Programa para la Transformación y el Fortalecimiento Académicos de las Escuelas Normales. Plan de estudios. México: SEP.

Secretaría de Educación Pública (2006). Libro de matemáticas segundo grado. México: SEP.

Secretaría de Educación Pública (2011). Planes y programas de estudio 2011. Educación Básica. México: SEP.

Steinbring, H. (2005). The Construction of new Mathematical Knowledge in Classroom Interaction. USA: Springer.

Tversky, A. y Kahneman, D. (1982). Availability: A heuristic for judging frequency and probability. En D. Kahneman, P. Slovic y A. Tversky (Eds.), Judgment under uncertainty: Heuristics and biases. EEUU: University Press.

Vygotski, L. S. (1997). Fundamentos de la Defectología. Obra Escogidas V. España: Visor Dis. 